\title{
Observation of a one-dimensional spin-orbit gap in a quantum wire
}

\author{
C. H. L. Quay ${ }^{1,2 \star}$, T. L. Hughes ${ }^{1 \dagger}$, J. A. Sulpizio', L. N. Pfeiffer ${ }^{2 \dagger}$, K. W. Baldwin ${ }^{2 \dagger}$, K. W. West ${ }^{2 \dagger}$, \\ D. Goldhaber-Gordon ${ }^{1}$ and R. de Picciotto ${ }^{2 \dagger}$
}

\begin{abstract}
Understanding the flow of spins in magnetic layered structures has resulted in an increase in data storage density in hard drives over the past decade of more than two orders of magnitude'. Following this remarkable success, the field of 'spintronics' or spin-based electronics ${ }^{1-3}$ is moving beyond effects based on local spin polarization and is turning towards spin-orbit interaction (SOI) effects, which hold promise for the production, detection and manipulation of spin currents, allowing coherent transmission of information within a device ${ }^{1,2}$. Although SOI-induced spin transport effects have been observed in two- and three-dimensional samples, these have been subtle and elusive, often detected only indirectly in electrical transport or else with more sophisticated techniques $^{4-9}$. Here we present the first observation of a predicted 'spin-orbit gap' in a one-dimensional sample, where counter-propagating spins, constituting a spin current, are accompanied by a clear signal in the easily measured linear conductance of the system ${ }^{10,11}$. We first introduce the class of phenomena we dub 'the one-dimensional spin-orbit gap' using a simple example adapted from ref. 10, then describe our experiment in detail and finally present a more elaborate model that captures most of the features seen in our data.
\end{abstract}

The SOI is a relativistic effect where a charged particle moving in an electric field experiences an effective magnetic field, which couples to its $\operatorname{spin}^{12}$. In semiconductor heterostructures, the electric field can arise as a result of either the lack of an inversion centre in the crystal (bulk inversion asymmetry) or a lack of symmetry in an external confining potential (structural inversion asymmetry) resulting from crystal interfaces or other structures such as metallic gates ${ }^{13}$. The strength of the resulting effective magnetic field is proportional to both the particle's momentum and the original electric field.

Consider a spin-degenerate one-dimensional (1D) sub-band with a Hamiltonian $H_{0}=\hbar^{2} k^{2} / 2 m$, where $\hbar$ is the reduced Planck's constant, $k$ is the particle's momentum and $m$ is its mass (Fig. 1a). The leading-order spin-orbit contribution to the Hamiltonian is $H_{\mathrm{SO}}=\beta \boldsymbol{\sigma} \cdot(\mathbf{k} \times \nabla V)$, where $\boldsymbol{\sigma}$ is the particle's spin, $V$ is the electrostatic potential and $\beta$ is a material-dependent parameter ${ }^{14}$. This term breaks the spin degeneracy of the system and results in two spinful sub-bands separated by a lateral (wavevector) shift as shown in Fig. 1b.

Despite this rather striking change in the band structure, measurements of conductance through the system cannot distinguish the situation shown in Fig. $1 \mathrm{~b}$ from the case where the spins are degenerate. In both cases the edges of the two spin sub-bands occur at the same energy, so in both cases the conductance rises by $G_{0}=2 e^{2} / h$ when the Fermi level of the system is tuned through this energy (for example, by applying a voltage to a nearby gate) ${ }^{15-17}$ (Fig. 1d.) To detect the spin-orbit shift in a transport measurement, a different approach is needed.

Note that the spins as shown in Fig. $1 \mathrm{~b}$ are polarized in the direction of $\mathbf{B}_{\mathrm{SO}}$, which is perpendicular to both the momentum (that is, the 1D wire) and the external electric field. ( $\mathbf{B}_{\mathrm{SO}}$ can be anywhere in the $y-z$ plane in Fig. 2.) A magnetic field applied exactly along $\mathbf{B}_{\mathrm{SO}}$ shifts the spinful bands up and down by the Zeeman energy respectively, splitting each step of size $G_{0}$ into two steps of size $G_{0} / 2$ as shown in Fig. 1e. Here spin is polarized alternately up and down for the respective spinful bands and thus the charge current can be completely spin polarized when only one spinful band is occupied; however, charge transport measurements still reveal no difference between this system and one where there is no SOI.

Now consider a magnetic field applied perpendicular to $\mathbf{B}_{\mathrm{SO}}$ (say along the wire): the two spinful bands are mixed by this term so that the zero-field crossing point becomes an anticrossing. We call this feature the spin-orbit gap (Fig. 1c). When the Fermi level lies within such a gap, two Fermi points (for example, 3 and 4 in Fig. 1f) contribute to the current as opposed to the four at the same energy in Fig. $1 d$. The conductance is thus reduced by $G_{0} / 2$ as shown in Fig. If. In addition, the current from this sub-band is completely spin polarized in a way that is expected to be robust to moderate disorder: holes must scatter between the points 1 and 2 or between 3 and 4 (Fig. 1f) and undergo a spin flip to backscatter. In such a system of counter-propagating spins (very nearly what is termed a 'helical liquid'18), the direction of the spin current is expected to be independent of the sign of the voltage applied across the system and is determined only by the sign of the SOI. Intriguingly, a pure spin current, without charge current, is expected to exist at zero bias voltage.

Finally, a magnetic field applied neither exactly perpendicular nor parallel to $\mathbf{B}_{\mathrm{SO}}$ results in a mixture of the two effects described above ${ }^{10}$.

We turn now to our experiment. Our samples are GaAs/AlGaAs hole quantum wires produced by the cleaved-edge overgrowth method $^{19,20}$. Starting with an extremely high-mobility 2D hole gas (2DHG) realized in a carbon-doped AlGaAs/GaAs/AlGaAs quantum well ${ }^{21}$, the sample is cleaved and more AlGaAs is grown using molecular beam epitaxy over the freshly exposed surface. Further

\footnotetext{
${ }^{1}$ Physics Department, Stanford University, Stanford, California 94305-4060, USA, ${ }^{2}$ Bell Labs, Alcatel Lucent, Murray Hill, New Jersey 07974, USA. †Present addresses: Laboratoire de Physique des Solides (CNRS UMR 8502), Bâtiment 510, Université Paris-Sud 11, 91405 Orsay, France (C.Q.H.L.); Physics Department, University of Illinois Urbana-Champaign, Urbana, Illinois 61801, USA (T.L.H.); Department of Electrical Engineering, Princeton University, Princeton, New Jersey 08544, USA (L.N.P., K.W.B., K.W.W.); B-Nano Ltd, 2 Meir Weisgal Road, Rehovot 76326, Israel (R.d.P.). *e-mail: cquayhl@stanfordalumni.org.
} 
a

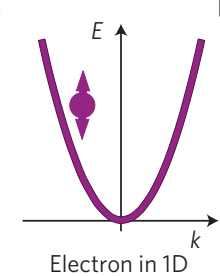

d

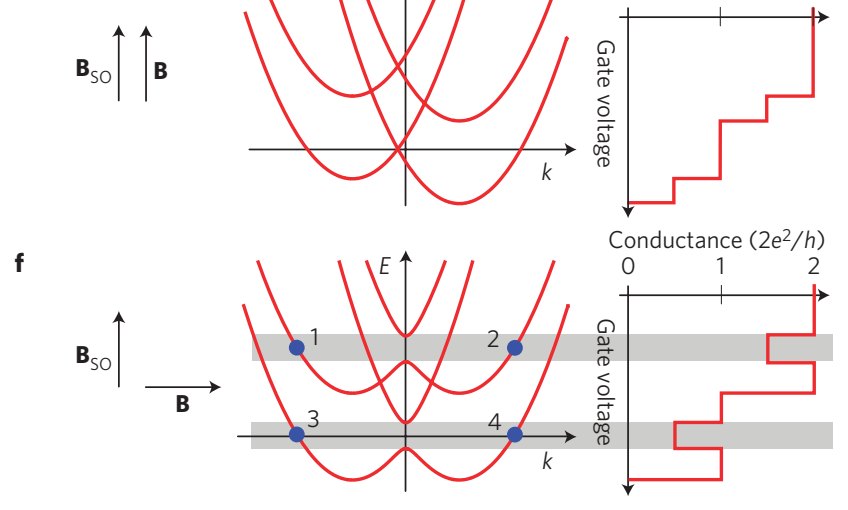

Figure 1 | The spin-orbit gap in a simple model and the associated conductance features. $\mathbf{a}$, The dispersion relation for electrons in a spin-degenerate 1D sub-band. $\mathbf{b}$, The SOl lifts the spin degeneracy, displacing spinful sub-bands laterally with respect to each other. c, An applied magnetic field can mix the two spin bands, creating the anticrossing we dub the spin-orbit gap. When the Fermi energy is tuned to be within this gap, particles of opposite spin travel in opposite directions, producing a spin current. At the same time, a clear drop is expected in the conductance, shown in f. d. With SOI but without an applied magnetic field the conductance of the system increases by a step of $2 e^{2} / \mathrm{h}$ each time the gate voltage aligns the Fermi energy to the bottom of a sub-band. e, A magnetic field applied parallel to $\mathbf{B}_{\mathrm{SO}}$ splits each step into two half-steps of $\mathrm{e}^{2} / h$. $\mathbf{f}$, With a magnetic field applied perpendicular to $\mathbf{B}_{\mathrm{SO}}$, spin-orbit gaps appear as in $\mathbf{c}$ and the conductance drops when the Fermi energy lies within such a gap.

carbon doping leads to hole accumulation at the GaAs/AlGaAs interface on the cleavage plane, resulting in a $1 \mathrm{D}$ wire (Fig. 2a). We are able to apply a magnetic field either parallel or perpendicular to the wire (in the $x$ or $y$ directions indicated in Fig. 2b).

The basic properties of our wires have been or will be reported in other publications ${ }^{20}$. Another body of work on quasi-1D wires in GaAs (ref. 22,23) focused on gate-defined quantum point contacts on a $2 \mathrm{DHG}$ grown in the 311 direction. These studies found that, because of the SOI, the effective $g$-factor depends strongly on the particular sub-band studied as well as the direction of the wire and that of the magnetic field with respect to the crystal axes. They did not, however, observe signs of the spin-orbit gap reported below, perhaps because of the growth direction of their $2 \mathrm{DHG}$ or their nearly symmetrical confinement potential. Recent tunnelling spectroscopy of carbon-nanotube quantum dots revealed few-electron states shaped by spin-orbit coupling, with a Hamiltonian and magnetic-field coupling rather different from that in semiconductor nanowires such as ours ${ }^{24}$. a

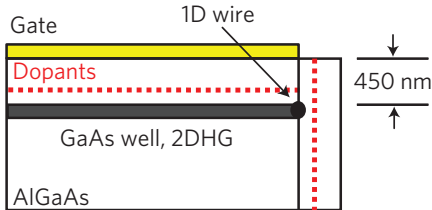

b

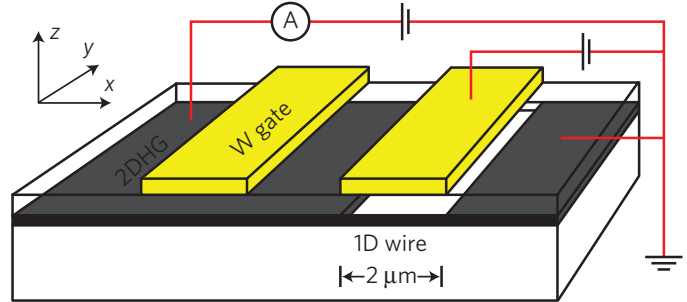

Figure 2 | The device and measurement set-up. a, Cross-section of our devices, which are fabricated by the cleaved-edge overgrowth method ${ }^{19,20}$ b. Measurement scheme for the 1D hole wires. A section of the wire is isolated using a gate, which depletes the $2 \mathrm{DHG}$ just beneath it. Conductance is measured using ohmic contacts to the $2 \mathrm{DHG}$ on either side of the wire and decreases in steps, each of which corresponds to the depletion of a 1D sub-band ${ }^{20}$.
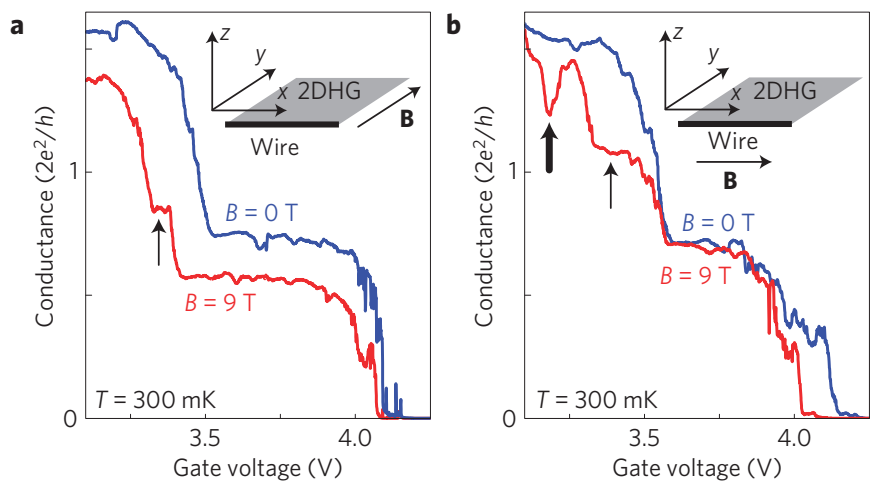

Figure 3 | The first two conductance steps of a quantum wire and their evolution in magnetic fields applied in two different directions. $a, A$ field perpendicular to the wire splits the second step at zero field (blue trace) into two half-steps, the first marked with an arrow (red trace). b. A field parallel to the wire produces a half-step (arrow) and a dip (thick arrow), signifying the presence of a spin-orbit gap. Both features are absent in the absence of a magnetic field (blue traces). On the first step (gate voltages larger than $3.5 \mathrm{~V}$ ) no dip is observed and half-steps, if they exist at all, are hardly discernible.

In Fig. $2 b$, applying a positive voltage to pre-fabricated gates on the top surface of the wafer, we deplete first the 2DHG under the gate to isolate the $1 \mathrm{D}$ wire and subsequently the sub-bands of the wire. The conductance, measured between ohmic contacts to the 2DHG on either side of the gate/wire, decreases in steps as the wire sub-bands are successively depleted. (Unlike in quantum point contacts, the conductance steps in these systems do not correspond to the ideal value of $2 e^{2} / h$ because of the imperfect coupling between the $2 \mathrm{DHG}$ and the $1 \mathrm{D}$ wire as described in greater detail in ref. 25.)

In Fig. 3-which shows our main experimental results-we focus on the first two conductance steps seen at zero magnetic field (blue traces) corresponding to the lowest two sub-bands in the wire, and their evolution in magnetic fields applied in two different directions, $y$ and $z$ (red traces). Concentrating first on the second, higher, conductance step, we see that a magnetic field in the $y$ direction transforms this step into two half-height steps (Fig. 3a), whereas a field along $x$ produces both two half-steps and a dip 


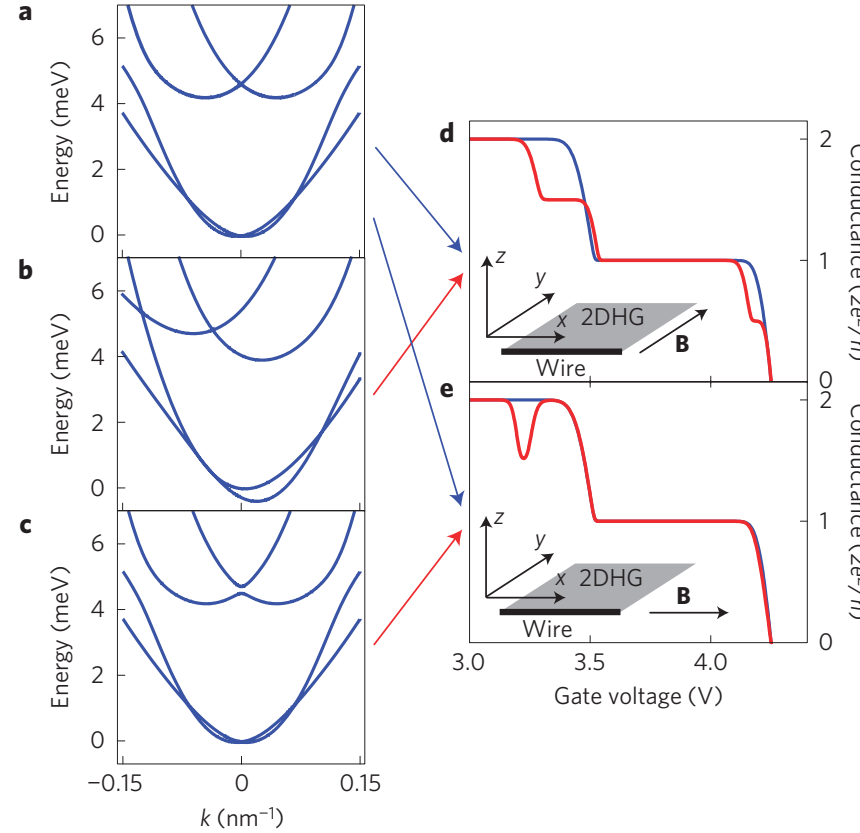

Figure 4 | Predictions from our model of band structures and

conductance traces. a, The dispersion relations of the two lowest quantum wire sub-bands with no applied magnetic field. $\mathbf{b}$, The same bands in a magnetic field of $9 \mathrm{~T}$ perpendicular to the wire, with $g=2$. $\mathbf{c}$, The same bands in a magnetic field of $9 \mathrm{~T}$ parallel to the wire, with $g=2 / 9$. $\mathbf{d}$, Conductance traces calculated from $\mathbf{a}$ (blue) and $\mathbf{b}$ (red) with the experiment temperature of $300 \mathrm{mK}$. Compare to Fig. 3a. e, Conductance traces calculated from $\mathbf{a}$ (blue) and $\mathbf{c}$ (red) with the experiment temperature of $300 \mathrm{mK}$. Compare to Fig. 3b.

(Fig. 3b). In contrast, the first step seems unaffected by magnetic field in either direction.

Let us try to understand these results in terms of the simple model presented in Fig. 1. Assuming that the main contribution to the spin-orbit effect results from structural inversion asymmetry, we expect $\mathbf{B}_{\mathrm{SO}}$ to be perpendicular to the wire, in the $y-z$ plane, as the structure is translationally invariant along the length of the wire. Thus, a magnetic field in the $x$ direction (perpendicular to $\mathbf{B}_{\mathrm{SO}}$ ) should produce dips in the zero-field conductance steps (as in Fig. 1f), whereas one in the $y$ direction should split each zero-field conductance step into two half-steps (as in Fig. 1e) and possibly also produce dips simultaneously.

Our data bear more than a passing resemblance to the expectations from the simple model, yet the two are significantly different in their details. A more realistic model, described below, enables us to more fully understand our results.

We use a four-band Luttinger model ${ }^{13}$ (not to be confused with the Tomonaga-Luttinger liquid theory) and include confinement in two directions, taking into account the orientation of our wire in the GaAs crystal. This model considers the lowest four spinful sub-bands, with the higher sub-bands being ignored. The parameters in the model are: $C, \gamma_{1}, \gamma_{2}, \gamma_{3}, \chi, d_{y}, d_{z}$, $r_{y}$ and $r_{z} . C$ and the $\gamma_{i}$ are bulk (Al)GaAs band structure parameters that are well established in the literature. $\chi$ describes the leakage of the 1D hole wavefunctions from the GaAs where they are nominally confined into the surrounding AlGaAs; however, the model is not very sensitive to this parameter. $d_{y}$ and $d_{z}$ describe the confinement in the $y$ and $z$ directions, and $r_{y}$ and $r_{z}$ are the strength of the structural-inversion-asymmetry electric field in the $y$ and $z$ directions. We start by using an isotropic value of 2 for the $g$-factor and return to this point later. The full Hamiltonian and details of its derivation as

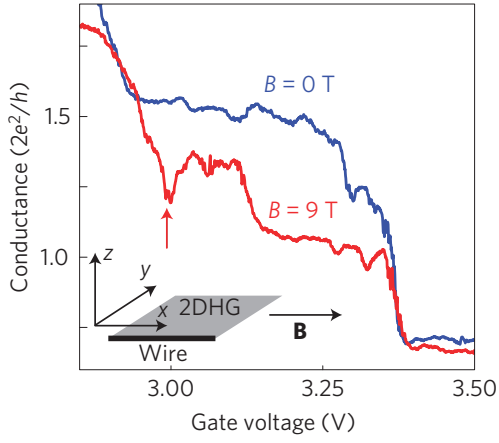

Figure $\mathbf{5}$ | Reproducibility. Data from a separate device, showing the dip on the second step resulting from the presence of the spin-orbit gap.

well as values chosen for each parameter can be found in the Supplementary Information.

Figure 4a shows the lowest energy bands calculated with this Hamiltonian in the absence of magnetic field. Focusing first on the upper pair of bands, we see that applying a field in the $y$ direction shifts them in energy (Fig. 4b), producing a double step in the conductance trace (Fig. 4d), which is seen in our data (Fig. 3a). In contrast, a field in the $x$ direction produces a gap in the band structure (Fig. 4c) and a dip in the conductance trace (Fig. 4e), also seen in our data (Fig. 3b).

As for the lower pair of bands, the spin splitting at $B=0$ is much smaller than in the upper bands. Furthermore, as in our data, magnetic fields in both directions do not have much of an effect (Figs 3 and 4). (The small half-step in the red trace in Fig. $4 \mathrm{~d}$ could be obscured in the data by the disorder-related features near depletion.)

Clearly the coupling between the two lower bands resulting from the SOI, or a magnetic field, is suppressed. This is a consequence of the fact that these bands have been chosen in the model to be primarily of heavy-hole character-their carriers have spin $\pm 3 / 2$ a choice motivated by our data and other physical considerations as explicated in the Supplementary Information. The leading-order Rashba and Zeeman terms are linear order in spin operators and thus can couple only states with $\Delta S=1$. As $\Delta S=3$ for the heavy holes, the two lower sub-bands are not coupled by these terms. Their coupling requires cubic-order terms in the spin operators. Such terms, however, are small in all components of the Hamiltonian: Rashba and Zeeman couplings that are cubic in spin operators do exist, but their coupling constants are suppressed ${ }^{13}$. The lowest order Dresselhaus term is cubic in spin operators, but in GaAs it is again very small ${ }^{13}$ and thus has almost no effect on the bands.

Indeed, the Dresselhaus term has a minimal effect not only on the lower bands but on the upper pair of bands as well. Therefore, the spin-orbit effects seen in our samples come primarily from the asymmetry of the confining potential of the $1 \mathrm{D}$ wire, that is, the Rashba term. We find further that the electric field associated with this potential is stronger in the $z$ direction than in the $y$ direction.

We return now to the question of the $g$-factor. We find that, to produce features of the experimentally observed sizes at the applied $9 \mathrm{~T}$ field, our model requires an effective $g$-factor of two in the $y$ direction and an effective $g$-factor of $2 / 9$ in the $x$ direction (Fig. 4 d,e). Such an anisotropy in the effective $g$-factor is expected in the presence of spin-orbit coupling and is dependent on the strength and direction of confinement ${ }^{13,22,23}$.

(One must be wary when speaking of effective $g$-factors in the presence of spin-orbit coupling as quantities commonly interpreted as 'spin splittings' are not necessarily equal to $g^{*} \mu_{\mathrm{B}} B$, where $g^{*}$ is the effective $g$-factor in the Zeeman term in the Hamiltonian. For example, in the simple 1D model described in Fig. 1, the situation in Fig. If could be interpreted as $g^{*}=0$ on the basis of measurements 
of sub-band edges such as those in refs 25 and 26, whereas $g^{*}$ in the model is most definitely finite.)

We are thus able to describe most of the important features of our data with the proposed model and to identify their physical causes. Further theoretical work is needed to understand one feature of our data that our model fails to capture: the occurrence of a double step in addition to a dip when the magnetic field is applied in the $x$ direction.

We also acquired data similar to those in Fig. 3 at finely spaced intermediate fields (Supplementary Information). These data show that the observed conductance dip and half-steps develop gradually with magnetic field, enabling us to rule out resonances associated with particular values of the magnetic field as the cause of the observed features. In addition, these features - and in particular the conductance dip-were observed in a separate device (Fig. 5) and persist after thermal cycling; thus, they are also not linked to particular configurations of the disorder potential in the device.

We have observed for the first time the spin-orbit gap predicted for 1D systems and we have developed a model that describes most of the detailed features of our data by taking into account the materials properties of $(\mathrm{Al}) \mathrm{GaAs}$.

Several directions seem promising for the further exploration of these gaps. For this particular device geometry, even cleaner wires would allow the study of spin-orbit effects at finite bias voltage and in higher sub-bands. A tunnelling set-up such as in ref. 26 could probe the dispersion relations directly; and a side gate as in ref. 27 would allow tuning the strength of the electric field that gives rise to the spin-orbit effect.

For the purposes of spintronics applications, verification of spin transport, particularly at zero bias, would be of great interest. This could be achieved either through direct detection of the spin current or through the detection of spin accumulation at the two ends of the device. It would also be of practical interest to explore the possibility of producing such spin-orbit gaps in the lowest sub-band and without a magnetic field. Although in our experiments the spin-orbit gap is induced by a magnetic field, this may not be necessary: the two spin bands could be mixed at $k=0$ in other ways, perhaps through the use of materials with magnetic order or through controlled doping with magnetic impurities.

Finally, in other studies Coulomb interactions have been found to have marked effects in 1D systems including cleaved-edge overgrowth electron wires ${ }^{28}$, and further work is necessary to understand their role in these newly developed systems.

\section{Received 23 November 2009; accepted 16 February 2010;} published online 21 March 2010

\section{References}

1. Fert, A. Nobel lecture: Origin, development, and future of spintronics. Rev. Mod. Phys. 80, 1517-1530 (2008)

2. Bratkovsky, A. M. Spintronic effects in metallic, semiconductor, metal-oxide and metal-semiconductor heterostructures. Rep. Prog. Phys. 71, 026502 (2008).

3. Žutić, I., Fabian, J. \& Das Sarma, S. Spintronics: Fundamentals and applications. Rev. Mod. Phys. 76, 323-410 (2004).

4. Kato, Y. K., Myers, R. C., Gossard, A. C. \& Awschalom, D. D. Observation of the spin Hall effect in semiconductors. Science 306, 1910-1913 (2004).

5. Sih, V. et al. Generating spin currents in semiconductors with the spin Hall effect. Phys. Rev. Lett. 97, 096605 (2006)

6. König, M. et al. Quantum spin Hall insulator state in HgTe quantum wells. Science 318, 766-770 (2007).

7. Frolov, S. M. et al. Ballistic spin resonance. Nature 458, 868-871 (2009).
8. Chen, Y. L. et al. Experimental realization of a three-dimensional topological insulator, $\mathrm{Bi}_{2} \mathrm{Te}_{3}$. Science 325, 178-181 (2009).

9. Xia, Y. et al. Observation of a large-gap topological-insulator class with a single Dirac cone on the surface. Nature Phys. 5, 398-402 (2009).

10. Pershin, Y. V., Nesteroff, J. A. \& Privman, V. Effect of spin-orbit interaction and in-plane magnetic field on the conductance of a quasi-one-dimensional system. Phys. Rev. B 69, 121306(R) (2004).

11. Zhang, S., Liang, R., Zhang, E., Zhang, L. \& Liu, Y. Magnetosubbands of semiconductor quantum wires with Rashba and Dresselhaus spin-orbit coupling. Phys. Rev. B 73, 155316 (2006).

12. Griffiths, D. J. Introduction to Electrodynamics 3rd edn (Prentice Hall, 1998).

13. Winkler, R. Spin-Orbit Coupling Effects in Two-Dimensional Electron and Hole Systems Vol. 191 (Springer Tracts in Modern Physics, Springer, 2003).

14. Elliott, R. J. Theory of the effect of spin-orbit coupling on magnetic resonance in some semiconductors. Phys. Rev. 96, 266-279 (1954).

15. Cronenwett, S. M. Coherence, Charging and Spin Effects in Quantum Dots and Point Contacts. PhD thesis, Stanford Univ. (2001).

16. Kouwenhoven, L. P. et al. Nonlinear conductance of quantum point contacts. Phys. Rev. B 39, 8040-8043 (1989).

17. Glazman, L. I. \& Khaetskii, A. V. Nonlinear quantum conductance of a point contact. JETP Lett. 48, 591-595 (1998).

18. Wu, C., Bernevig, B. A. \& Zhang, S.-C. Helical liquid and the edge of quantum spin Hall systems. Phys. Rev. Lett. 96, 106401 (2006).

19. Pfeiffer, L. et al. Formation of a high quality two-dimensional electron gas on cleaved GaAs. Appl. Phys. Lett. 56, 1697-1699 (1990).

20. Pfeiffer, L. N., de Picciotto, R., Quay, C. H. L., West, K. W. \& Baldwin, K. W. Ballistic hole transport in a quantum wire. Appl. Phys. Lett. 87, 073111 (2005)

21. Manfra, M., Pfeiffer, L., West, K., de Picciotto, R. \& Baldwin, K. High mobility two-dimensional hole system in GaAs/AlGaAs quantum wells grown on (100) GaAs substrates. Appl. Phys. Lett. 86, 162106 (2005).

22. Klochan, O. et al. The interplay between one-dimensional confinement and two-dimensional crystallographic anisotropy effects in ballistic hole quantum wires. New J. Phys. 11, 043018 (2009).

23. Danneau, R. et al. Zeeman splitting in ballistic hole quantum wires. Phys. Rev. Lett. 97, 026403 (2006).

24. Kuemmeth, F., Ilani, S., Ralph, D. C. \& McEuen, P. L. Coupling of spin and orbital motion of electrons in carbon nanotubes. Nature 452, 448-452 (2008).

25. de Picciotto, R. et al. 2D-1D coupling in cleaved edge overgrowth. Phys. Rev. Lett. 85, 1730-1733 (2000).

26. Auslaender, O. et al. Tunneling spectroscopy of the elementary excitations in a one-dimensional wire. Science 295, 825-828 (2002).

27. Yacoby, A., Stormer, H. L., Baldwin, K. W., Pfeiffer, L. N. \& West, K. W. Magneto-transport spectroscopy on a quantum wire. Solid State Commun. 101, 77-1 (1997)

28. Auslaender, O. M. et al. Spin-charge separation and localization in one dimension. Science 208, 88-92 (2005).

\section{Acknowledgements}

We thank P. Joyez, M. A. Wistey, J. E. Moore and A. S. Goldhaber for helpful discussions and/or comments on the manuscript. J.A.S. acknowledges support from a National Science Foundation graduate fellowship, C.Q.H.L. support from a Harvey Fellowship and Bell Labs, and D.G.-G. a Fellowship from the David and Lucile Packard Foundation and a Hellman Faculty Scholar Award. Work at Stanford was primarily supported by the AFOSR under contracts FA9550-04-1-0384 (PECASE) and FA9550-08-1-0427.

\section{Author contributions}

K.W.W. and L.N.P. carried out the molecular beam epitaxy growth, R.d.P. and C.Q.H.L. the rest of the sample fabrication. K.W.B. characterized the 2DHGs before the cleave as well as control samples from the overgrowth step. R.d.P., C.Q.H.L. and J.A.S. carried out measurements. T.L.H. derived the theoretical model with input from R.d.P. and C.Q.H.L. C.Q.H.L., R.d.P., D.G.-G., T.L.H. and J.A.S. analysed the data. C.Q.H.L. wrote the manuscript together with R.d.P., D.G.G. and T.L.H. R.d.P. and L.N.P. had the idea for the experiment

\section{Additional information}

The authors declare no competing financial interests. Supplementary information accompanies this paper on www.nature.com/naturephysics. Reprints and permissions information is available online at http://npg.nature.com/reprintsandpermissions. Correspondence and requests for materials should be addressed to C.Q.H.L. 